Apidologie, 1987, 18 (3), 279-288

\title{
WELCHE DIÄT ERGIBT ARBEITERINNEN BEI IN VITRO-AUFZUCHT VON HONIGBIENEN ? ${ }^{(*)}$
}

\author{
Dieter WITTMANN und Wolf ENGELS \\ Lehrstuhl Entwicklungsphysiologie, Institut für Biologie III (Zoologie) der Universität Tübingen, \\ Auf der Morgenstelle 28, D 7400 Tübingen
}

\section{ZUSAMMENFASSUNG}

Verschiedene halbsynthetische Diäten wurden auf ihre Eignung für eine in vitro-Aufzucht von Arbeiterinnen der Honigbiene (Apis mellifica) geprüft. Dabei wurden in Anlehnung an die Larvenernährung im Bienenvolk Diätprogramme mit unterschiedlicher Junglarven- und Altlarven-Diät konzipiert. 2 der 15 getesteten Diätprogramme ergaben bei Aufzuchtraten von ca 35-50\% eine Differenzierung von über $90 \%$ der Larven zu Arbeiterinnen. Die Ergebnisse werden auch in Hinblick auf die trophogene Basis der Kasten-Differenzierung diskutiert.

\section{EINLEITUNG}

Vor fast 100 Jahren wurden die ersten Untersuchungen über Kastenspezifische larvale Nahrungsfaktoren bei der Honigbiene veröffentlicht (Planta, 1888; Perez, 1889). Seither ist über diesen Problemkreis eine umfangreiche Literatur entstanden, die kürzlich von BeETSMA (1979) sowie DE WiLDE und BEETSMA (1982) zusammenfassend dargestellt wurde. Wesentliche Einsichten verdanken wir den in den letzten 25 Jahren entwickelten Verfahren zur in vitro-Aufzucht von Bienenlarven (vgl. HaNSER, 1980). Sie erlauben es, den Effekt bestimmter Diätkomponenten auf die Kasten-Differenzierung zu prüfen (RemBold, 1976). Die nur schwer kontrollierbaren Pflegemaßnahmen der Ammen sind dabei ausgeschaltet.

Die bisherigen Untersuchungen zum Kasten-Syndrom bei Honigbienen konzentrierten sich auf die Frage, welche Ernährungsbedingungen die Entwicklung einer weiblichen Larve zur Königin steuern. Unter welchen Voraussetzungen eine Arbeiterin entsteht, wurde kaum analysiert. Wir prüften, mit welchen

$\left(^{*}\right)$ Mit dankenswerter Förderung durch das Ministerium für Ernährung, Landwirtschaft, Umwelt und Forsten Baden-Württemberg, Stuttgart. 
Diäten in vitro Bienenarbeiterinnen aufgezogen werden können. Da im Volk Drohnen- und Arbeiterinnen-Larven vom 4. Stadium ab mit Pollen und Honig versetztes Futter erhalten, untersuchten wir speziell den Effekt von AltlarvenDiäten.

\section{MATERIAL UND METHODEN}

Zur in vitro-Aufzucht wurden ca $24 \mathrm{~h}$ alte Bienenlarven von Arbeiterinnen-Brutwaben aus Laborvölkern von Apis mellifica carnica in Fingerhüte umgelarvt. Die Junglarven wurden bei $34{ }^{\circ} \mathrm{C} \pm 0,4{ }^{\circ} \mathrm{C}$ und ca. $95 \%$ Luftfeuchtigkeit im Brutschrank gehalten und täglich 2 mal mit $0,2 \mathrm{ml}$ halbsyntheticher Diät versorgt, was eine ad libidum-Fütterung bedeutet. Die Junglarven-Diät bestand gewichtsmäßig zu $50 \%$ aus nativem Gelée royale ( $1 \mathrm{~kg}$-Mengen der Fa. H. Mack, Illertissen, wurden homogenisiert und dann portionsweise bis zur Verwendung innerhalb einiger Monate tiefgefroren gelagert). Außerdem enthielt sie $36,25 \%$ aqua bidest, 6,25\% Glucose, 6,25\% Fructose und 1,25\% Hefeextrakt (Difco bactoyeast extract, B 127, vgl. RemBold und LACKNER, 1981).

Vom 4. Larvenstadium an wurden Altlarven-Diäten verfüttert, bei denen der Hefeextrakt durch Wasser ersetzt war. Vor Diätwechsel sowie nach Einstellen der Nahrungsaufnahme am 6 . Tag der Larvenentwicklung wurden die Futterreste mit einer Wasserstrahlpumpe abgesaugt. Vom Streckmadenbis zum Imaginal-Stadium wurde eine Luftfeuchtigkeit von $65 \%$ eingestellt. (Herrn Prof. Dr. REMBOLD und Mitarbeitern danken wir für die Einführung in die in vitro-Technik der Aufzucht von HonigbienenLarven).

Ein Testansatz bestand jeweils aus 60 Larven. In 11 Versuchsserien mit je 2 Testansätzen wurden Altlarven-Diäten verfüttert, bei denen jeweils der Gelée royale-Anteil um $1 \%$ reduziert (49\%-40\% bzw. $35 \%$ ) und dafür der aqua bidest-Gehalt um $1 \%$ erhöht war. In 2 weiteren Versuchsserien mit je 2 Testansätzen wurden Altlarven-Diäten geprüft, bei denen $50 \%$ bzw. $100 \%$ des Gelée royale vorher 10 min. auf $60^{\circ} \mathrm{C}$ erhitzt worden waren. Schließlich wurde in 2 Versuchsserien mit 8 bzw. 10 Testansätzen an Jung- wie auch an Altlarven durchgehend die hefehaltige Junglarven-Diät bzw. hefefreie AltlarvenDiät verfüttert.

Als Aufzuchtrate wird der Prozentsatz an erhaltenen Imagines bezeichnet. Aufgrund ihrer Mandibelzähnung und der Behaarung der Hinterbein-Basitarsen wurden sie als Königin $q$, Intermediär-Formen \{ oder Arbeiterin $\not \subset$ klassifiziert (vgl. Rembold, 1976 ; WeIss, 1978).

\section{ERGEBNISSE}

\section{Beeinflußt der Gelée royale-Gehalt einer Altlarven-Diät die Aufzuchtraten?}

Wird in einer Altlarven-Diät der Anteil des Gelée royale schrittweise von 50 auf $40 \%$ vermindert und stattdessen mehr Wasser hinzugegeben, so sinkt die Aufzuchtrate von $70-80 \%$ auf etwa $30-40 \%$ ab (Abb. 1a). Bei $35 \%$ Gelée royale überleben nur ca. $20 \%$ der Bienen, ein noch geringerer Anteil bedingt eine fast $100 \%$ ige Mortalität.

Wie aus Abb. 1a ersichtlich, streuen die Überlebensraten der Versuchsserien mit je 120 Larven erheblich. Vergleicht man einzelne Bereiche mit hohem (über $47 \%$ ), mittlerem ( $46-43 \%$ ) und niedrigem ( $42 \%$ oder weniger) Gelée 
royale-Gehalt der Diät, so ergeben sich durchschnittliche Überlebensraten von $66,4 \pm 9,9 \%, 53,7 \pm 10,8 \%$ und 30,8 $\pm 9,8 \%$. Von diesen Mittelwerten sind der 2. und 3. sowie der 1 . und 3. signifikant verschieden ( $t-T e s t, p=0,05)$.

Bei Altlarven-Diäten, die knapp zur Hälfte aus Königinnenfuttersaft bestehen, sind Gelée royale-Gehalt und Aufzuchtrate somit positiv korreliert.

\section{Beeinflußt der Gelée royale-Gehalt} einer Altlarven-Diät die Kasten-Differenzierung?

Werden auch die Altlarven mit einer Junglarven-Diät gefüttert, die zur Hälfte aus Gelée royale besteht und mit Hefeextrakt angereichert ist, so sind etwa $2 / 3$ der späteren Imagines reine Arbeiterinnen (Abb. 1b, gestrichelte Linie). Erhalten die Altlarven stattdessen eine hefefreie Diät mit variiertem Gelée royale-Gehalt, so entstehen mehr, nämlich $70-90 \%$ Arbeiterinnen (Abb. 1b).

Eine klare Korrelation zwischen dem Anteil des Gelée royale am AltlarvenFutter und dem Prozentsatz an entstehenden Arbeiterinnen ist jedoch nicht zu erkennen.

\section{Beeinflußt die Gelée royale-Qualität einer Altlarven-Diät die Kasten-Differenzierung?}

Wird bei einer Standard-Altlarven-Diät mit $50 \%$ Gelée royale-Gehalt dieser zuvor erhitzt, so erhöht das den Anteil an adulten Arbeiterinnen auf über $70 \%$. (Abb. 2a, vgl. Diät 1 mit 2 und 3). Ist die Hälfte des Gelée royale hitzebehandelt, nimmt der Arbeiterinnen-Prozentsatz nur geringfügig zu, die Königinnen- und Interkast-Quoten sinken leicht ab (Abb. 2a, Diät 2). Wird hingegen der gesamte Gelée royale erhitzt, so entstehen neben wenigen Intermediär-Formen ganz überwiegend Arbeiterinnen. Königinnen entwickeln sich auf einer solchen Diät nicht mehr (Abb. 2a, Diät 3).

Betrachtet man diese Ergebnisse (Abb. 2a) unter dem Gesichtspunkt, ob die geprüften Diäten mit unbehandeltem oder erhitztem Gelée royale sich vorzugsweise zur Aufzucht von Arbeiterinnen eignen, so kann dies über einen Vergleich der gemittelten Kastenanteile geschehen. Wir stellen daher die Quoten der mit den Diäten 1, 2 und 3 insgesamt erhaltenen Arbeiterinnen ( $(q)$ den Nicht-Arbeiterinnen $(q, \phi)$ gegenüber. Es ergeben sich $\bar{x}=79,3 \%$, $\mathrm{s}=11,9 \%$ gegen $\overline{\mathrm{x}}=20,7 \%, \mathrm{~s}=11,9 \%$, der Unterschied ist hoch signifikant.

Vergleicht man die Daten der Abb. 2a mit denen der Abb. 1b, so kommt man zu dem Schluß, daß die Qualität des an Altlarven verfütterten Gelée royale für die Kasten-Differenzierung entscheidender ist als die Quantität. 


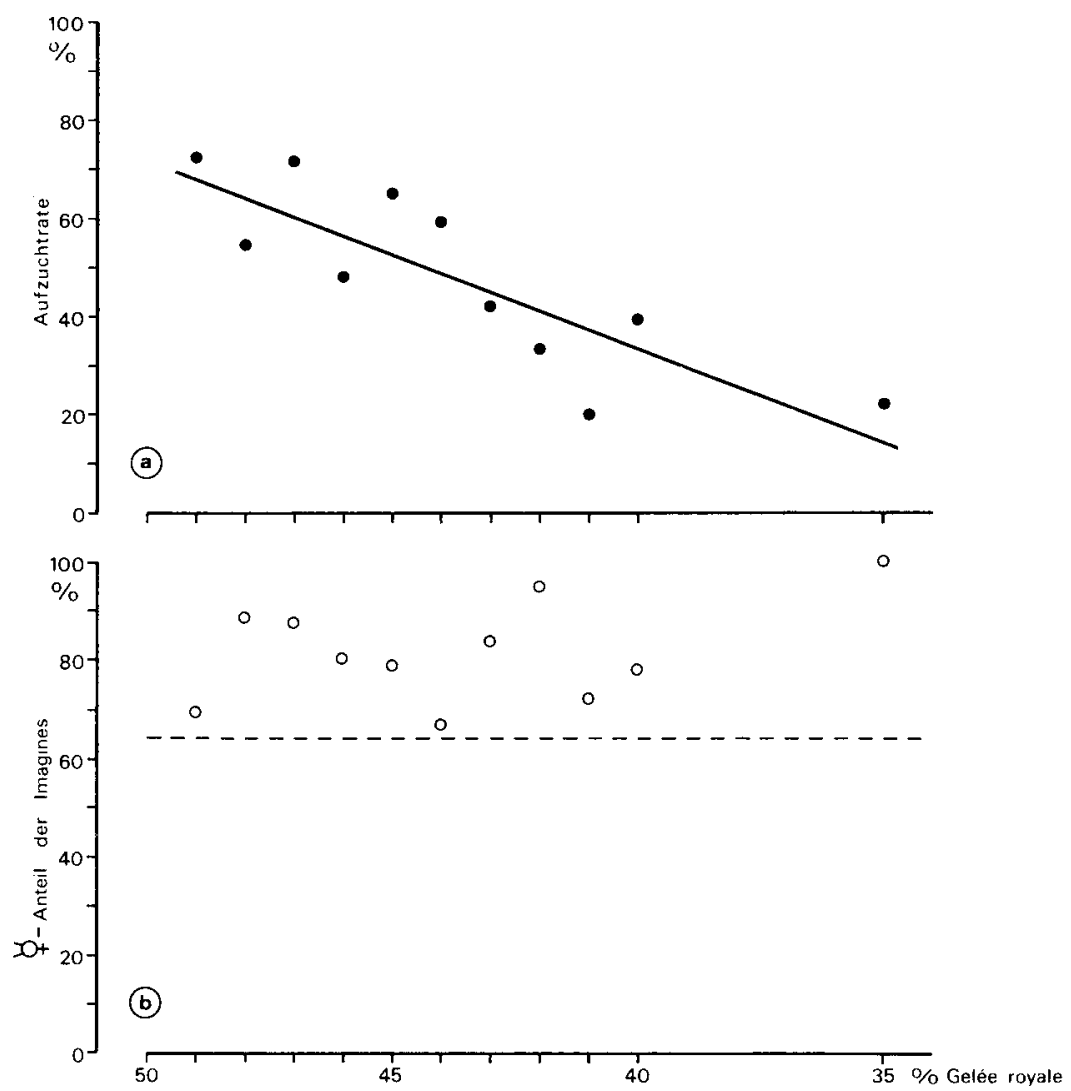

Авв. 1. - Einfluß des Gelée royale-Gehaltes der Altlarven-Diät

auf Aufzuchtrate (a) und Arbeiterinnen-Anteil (b) bei in vitro-Haltung von Honigbienen-Larven

Die waagerecht gestrichelte Linie in (b) gibt den Prozentsatz an Arbeiterinnen wieder, der in den Kontrollserien bei Verfüttern von Diät mit $50 \%$ Gelée royale und Hefeextrakt-Zusatz an Jung- und Altlarven erhalten wurde, vgl. dazu auch Abb. 2b, Diät 4.

FIG. 1. - Effect of royal jelly content in the diet on old honeybee larvae reared in vitro. (a) rate of survival and (b) percentage of adult workers

The dotted horizontal line in (b) represents the percentage of workers obtained in the control when young and old larvae were fed on a diet containing $50 \%$ royal jelly with yeast extract added. See also Fig. 2b, diet No. 4 .

\section{Welchen Einfluß hat Junglarven-Diät auf die Kasten-Differenzierung?}

Die Junglarven-Diät enthält wegen des Hefeextrakt-Zusatzes mehr Vitamine, Spurenelemente und andere essentielle Nahrungsfaktoren. Verfüttert man sie auch an die Altlarven, so entstehen zu rd. $65 \%$ Arbeiterinnen; Königinnen und Interkasten-Individuen stellen gut $10 \%$ bzw. $20 \%$ der Imagines (Abb. 2b, Diät 4). 

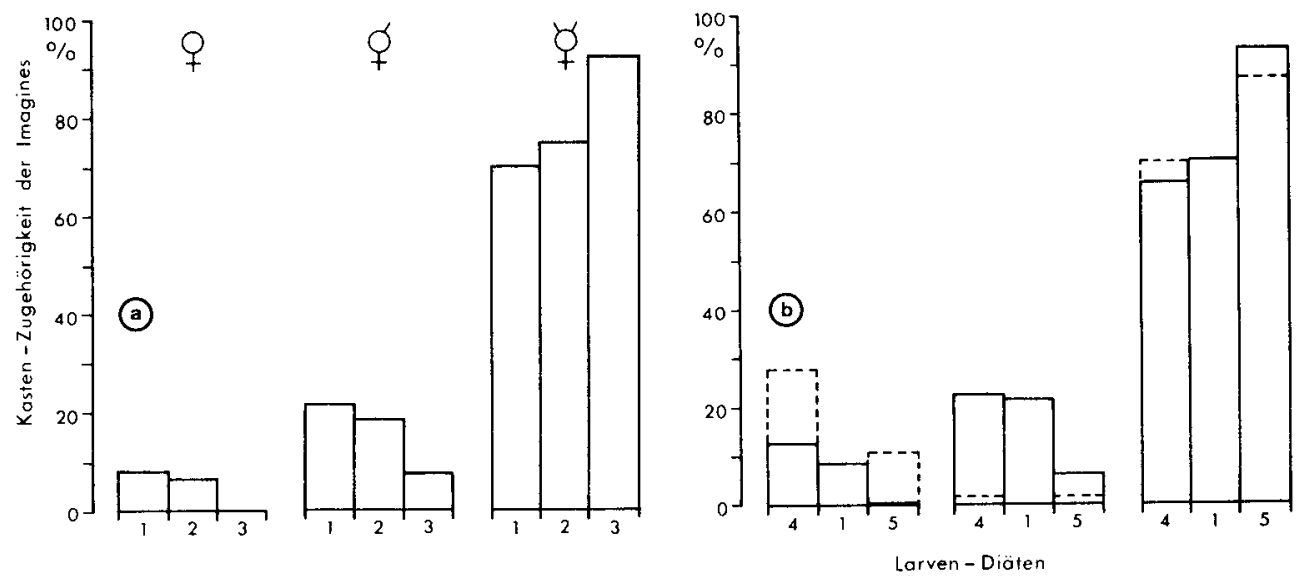

Aвв. 2. - Einfluß der Qualität einer Larven-Diät auf die Kasten-Differenzierung bei in vitro-Aufzucht von Honigbienen

(a) Effekt einer Hitzc-Bchandlung des Gelée royale in der Altlarven-Diät, (b) Effekt eines Hefeextrakt-Zusatzes zur Jung- und Altlarven-Diät. - (a) 1,2 und $3=$ Junglarven-Diät mit. Altlarven-Diät ohne Hefe-Extrakt ; in I $=0 \%, 2=50 \%, 3=100 \%$ des Gelée royale erhitzt. - (b) $4=$ Jung- und Altlarven-Diät mit Hefeextrakt. 1 = Junglarven-Diät mit, Altlarven-Diät ohne Hefeextrakt. $5=$ Jungund Altlarven-Diät ohne Hefecxtrakt. Mit den Diäten 3 und 5 wurden über $90 \%$ Arbeiterinnen erhalten. - Bci 4 und 5 sind außerdem die von Rembolo und Lackner (1981, dortige Fig. 2, A und B) mit gleichen Diäten erhaltenen Kasten-Prozentsätze gestrichelt cingezeichnet, sie stimmen mit unseren Daten gut übercin.

Fig. 2. - Effect of the quality of larval diet on caste differenciation of honeybees reared in vitro

(a) effect of heat treatment of the royal jelly content in the diet for old larvae. (b) effect of adding yeast extract to the diet for young and old larvae. - (a) 1,2 and $3=$ diet for young larvac with, for old larvac without yeast extract, $1=0 \%, 2=50 \%, 3=100 \%$ of the royal jelly heat treated. - (b) $4=$ dict for young and old larvac with yeast extract. 1 = dict for young larvae with, for old larvae without yeast extract. $5=$ diet for young and old larvae without yeast extract. - In 4 and 5 the dotted part of the histogram represents the very similar data obtained by REMBOLD and LACKNER (1981) using the same diets.

Wird der Hefeextrakt in der Altlarven-Diät weggelassen, erhöht dies den Arbeiterinnen-Anteil nur leicht. Es entwickeln sich weniger Königinnen bei etwa gleich viel Interkasten-Formen (Abb. 2b, Diät 1). Wenn auch die Junglarven-Diät ohne Hefeextrakt angesetzt wird, differenzieren sich nur noch ganz vereinzelt Larven zu Königinnen. Die Quote der Intermediär-Formen sinkt auf etwa $7 \%$ ab, und alle übrigen Imagines sind reine Arbeiterinnen (Abb. 2b, Diät 5).

Die Qualität der Junglarven-Diät ist also vor allem dann, wenn die Altlarven auf "magere " Kost gesetzt werden, ebenfalls von erheblicher Bedeutung für die Kasten-Differenzierung. 
Vergleicht man summarisch den Einfluß von Hefezusatz zum Larvenfutter auf die Kasten-Differenzierung (Abb. 2b), so können die Quoten der in je 10 Testansätzen mit den hefehaltigen Diäten 1 und 4 aufgezogenen Arbeiterinnen mit denen der hefefreien Diät 5 verglichen werden. Im Mittel waren dies $\overline{\mathrm{x}}=64,8 \%, \mathrm{~s}=29,3 \%$ gegenüber $\overline{\mathrm{x}}=93,0 \%, \mathrm{~s}=13,3 \%$. Die Arbeiterinnen-Anteile liegen, bei Aufzucht mit hefefreien Larvendiäten somit deutlich über denen mit hefehaltigen.

\section{Gibt es Kasten-Unterschiede in der Überlebensrate?}

Unter in vitro-Aufzucht-Bedingungen beeinflußt der Gelée royale-Gehalt der Altlarven-Diät die Überlebensrate bis zur Imago (Abb. 1a). Gibt es dabei hinsichtlich der Mortalität kastenspezifische Unterschiede? Wie aus Abb. 3 ersichtlich, ist dies tatsächlich der Fall, wenn auch in begrenztem Ausmaß. In Versuchsserien mit niedriger Aufzuchtrate überlebten relativ mehr Arbeiterinnen, bei hoher Aufzuchtrate dagegen mehr Interkasten-Formen und Königinnen.

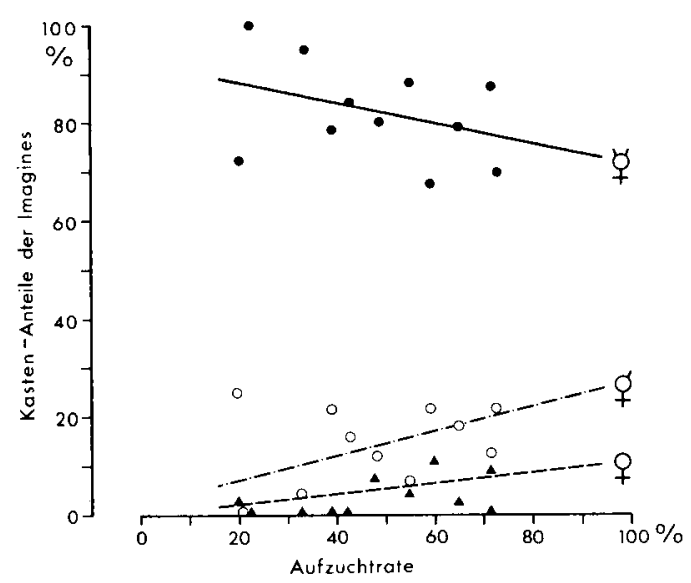

Aвв. 3. - Relation von Aufzuchtrate und Kasten-Zugehörigkeit bei in vitro-Entwicklung von Honigbienen-Larven

Die Daten der aus je 120 Larven bestehenden Diät-Serien mit variiertem Gelée royale-Gehalt in der Altlarven-Diät wurden hier nach Überlebensraten geordnet und in Kasten-Anteile aufgeschlüsselt. Die Einzelwerte streuen aufgrund der unterschiedlichen Diät-Bedingungen erheblich. Tendenziell lassen die Daten jedoch erkennen, daß bei günstigen Aufzuchtverhältnissen Königinnen und Interkasten-Formen relativ bessere Überlebenschancen haben, bei ungünstigen Bedingungen dagegen relativ mehr Arbeiterinnen überleben.

Fig. 3. - Relation of rate of survival and caste in honey bees reared in vitro

The data from the 120 larvae in test series in which diets for old larvae contained differing amounts of royal jelly were grouped according to rates of survival and split into caste percentages. There is some dispersion in the data according to the different dietary conditions. Nevertheless the following tendency may be recognized : under favorable rearing conditions queens and intercastes have a relatively better chance to survive. Unfavorable conditions, however, result in relatively more surviving workers. 
Unter ungünstigen Ernährungsumständen, insbesondere während der Altlarven-Periode, haben offenbar Arbeiterinnen die größten Überlebenschancen .

\section{Bewertung der Arbeiterinnen-Diät-Programme}

Der statistische Vergleich von mit verschiedenen Diäten erhaltenen Quoten an Arbeiterinnen gegenüber allen Nicht-Arbeiterinnen muß vor dem Hintergrund der von uns verwendeten Kasten-Klassifizierung gesehen werden. Innerhalb der morphologischen Bandbreite von reinen Arbeiterinnen bis zu reinen Königinnen wurden diesen beiden Klassen je $20 \%$ Variationsbreite, den Interkasten-Formen hingegen insgesamt $60 \%$ zugeteilt (vgl. REMBoLD, 1976). Wenn daher auf die Arbeiterinnen-Klasse mit $20 \%$ Anteil am morphologischen Variationsspektrum im Mittel 75-80\% und mit einzelnen Diäten über $90 \%$ der erhaltenen Imagines entfallen, können die entsprechenden DiätProgramme wohl als geeignete Aufzuchtverfahren für Arbeiterinnen angesehen werden.

\section{DISKUSSION}

\section{Technik der in vitro-Aufzucht von Arbeiterinnen}

Bislang stand keine definierte Diät für eine in vitro-Aufzucht von Honigbienen-Arbeiterinnen zur Verfügung. Mit 2 der 15 hier getesteten Diätprogramme gelang es, einen Arbeiterinnen-Anteil von über $90 \%$ der Imagines zu erhalten: Erstens bei Junglarven-Diät mit Hefeextrakt und Altlarven-Diät ohne Hefeextrakt, Gelée royale sämtlich erhitzt ; und zweitens bei Einheitsdiät ohne Hefeextrakt für Jung- und Altlarven. Beide Arbeiterinnen-Diät-Programme sind hinsichtlich ihres Effektes auf die Kasten-Differenzierung von recht ähnlicher Wirkung (vgl. Abb. 2a, Diät 3 und Abb. 2b, Diät 5). Das erste Programm ist von den Diät-Rezepturen und der Handhabung mit einem Futterwechsel ab L4 etwas umständlicher. Die Überlebensrate bis zur Imago lag für 120 getestete Larven bei $34 \%$. Das zweite ist mit Einheits-Diät nur aus Gelée royale, Glucose und Fructose für Jung- und Altlarven leicht anzuwenden, die Aufzuchtrate von $47 \%$ bei 600 Larven war noch besser.

Mit dem hier vorgestellten in vitro-Aufzuchtverfahren können also auf einfach herstellbarem halbsynthetischen Larvenfutter Bienen-Arbeiterinnen aller Entwicklungsstadien im Labor unter kontrollierten Bedingungen gehalten werden. Damit sind für viele Anwendungsbereiche die technischen Voraussetzungen gegeben. Es seien hier nur der Apis-Larven-Test zur Toxizitätsprüfung (Wittmann und Engels, 1981 ; Wittmann et al., 1987) oder eine in vitroZucht von Brutparasiten wie der Varroa-Milbe angeführt. 


\title{
2. Qualität versus Quantität in der trophogenen Basis der Kasten-Entwicklung
}

In unseren Versuchsserien erhielten alle Larven die gleichen Futtermengen, im Prinzip wurden sie ad libidum versorgt. Wie dargestellt, erwies sich für die Kasten-Entwicklung die Qualität der Larvennahrung als entscheidend. Zum selben Schluß kamen Rembold und LACKNer (1981) ; sie prüften in einem in vitro-Aufzucht-Experiment mit denselben Diäten wie wir die Wirkung von Hefeextrakt (vgl. Abb. 2b). Wird die Qualität einer halbsynthetischen Diät niedrig eingestellt, so kann sich eine weibliche Honigbienenlarve nicht mehr königinnengemäß differenzieren. Es entstehen Arbeiterinnen (vgl. EnGELs, 1987).

Überträgt man diese experimentellen Befunden auf die natürlichen Verhältnisse, so bedeutet es, daß im Bienenvolk für die massenweise Erzeugung von Arbeiterinnen offensichtlich nur ein minimaler Aufwand an Nahrungsqualität investiert werden muß. Ein solcher Fütterungsmodus resultiert in Individuen mit rudimentierten Reproduktionsorganen (vgl. WeIss, 1978 ; DE WILDE und BeEtsma, 1982), z.B. aber einer wohlausgeprägten Differenzierung des Gehirns (Lucht-Bertram, 1962). Die Größe einer Arbeiterin hängt dagegen von der aufgenommenen Futterquantität $a b$ und rangiert normalerweise im unteren Bereich der genetisch fixierten Variationsbreite weiblicher Bienen (BUCHNER, 1953).

In der Evolution der eusozialen Honigbienen entstand offensichtlich nicht eine spezifische Königinnen-Diät, für die früher ein Determinator postuliert wurde (BUTENANDT, 1959), sondern vielmehr ein qualifativ wie quantitativ balanciertes Minimal-Diätprogramm für Arbeiterinnen. Jede Steigerung der Qualität der Larvennahrung resultiert in einer Verschiebung der Differenzierung in Richtung Königin.

Eingegangen im August 1986. Angenommen im April 1987.

\author{
RÉSUMÉ \\ QUEL RÉGIME ALIMENTAIRE PERMET L'ÉLEVAGE IN VITRO \\ D'OUVRIËRES D'ABEILLES?
}

La base trophogénique de la différenciation des castes a été étudiée chez l'abeille en tenant compte principalement des facteurs induisant la reine. A ce jour les conditions alimentaires nécessaires au développement de l'ouvrière n'ont pas été analysées. Nous avons donc utilisé les techniques in vitro décrites par Hanser (1980) et par Rembold et Lackner (1981) pour tester l'action de 15 régimes semiartificiels sur la différenciation des castes. Le but était d'étudier les conditions nutritives pour élever des larves d'abeilles in vitro jusqu'à l'ouvrière adulte. Puisque les nourrices alimentent les $4^{\mathrm{e}}$ et $5^{\mathrm{e}}$ stades larvaires d'ouvrières et de mâles avec de la gelée mélangée à du miel et du pollen, nous avons utilisé des régimes différents pour les larves jeunes (L2 et L3) et les larves âgées (L4 et L5). 
Le régime pour les jeunes larves était constitué d'un mćlange de 50,00\% de gelée royale, 36,25\% d'aqua bidest, $6,25 \%$ de fructose, $6,24 \%$ de glucose et $1,25 \%$ d'extrait de levure (Difco bacto-yeast extract). Dans le régime pour les larves âgées, l'extrait de levure était remplacé par de l'eau.

Une réduction progressive de la tencur en gelée royale du régime pour les larves âgées a provoqué une augmentation de la mortalité. On n'a pas observé d'action significative sur la différenciation des castes (Fig. 1). Le traitement thermique de la gelée royale a inhibé la production de reines mais un plus grand nombre d'ouvrières a été ćlcvé avec ce régime (Fig. 2a). On a obtenu des résultats similaires en nourrissant les larves avec un régime dépourvu d'extrait de levure (Fig. 2b). Ceci indique que la qualité de la nourriture a une plus grande influence sur le développement des castes que la quantité.

Une méthode simple et efficace pour produire principalement (plus de $90 \%$ ) des ouvrières pures consiste à nourrir uniformément les larves de tous stades avec un régime dépourvu d'extrait de levure. La production d'abeilles adultes a été de $50 \%$ environ. On peut en particulier utiliser ce programme de nourrissement pour étudier in vitro les effets des pesticides sur le développement larvaire aussi bien que les parasites du couvain, tel l'acarien Varroa.

On conclut de ces expériences que chez les abeilles hautement eusociales un programme de nourrissement strictement équilibré ne s'est développé que pour les ouvrières. Ceci permet à la colonie de produire de grands nombres d'individus non fertiles avec une nourriture de qualité minimum. L'élevage en masse des ouvrières devient ainsi efficace avec un investissement relativement faible. Toute amélioration de la qualité fait basculer la différenciation des castes vers les reines.

\section{SUMMARY}

\section{ON WHICH DIET CAN WORKER HONEYBEES BE REARED IN VITRO?}

The trophogenic basis of caste development has been studied in honeybee larvae mainly with respect to queen inducing factors. To date the alimentary conditions for worker development have not been analysed. Therefore we used in vitro techniques described by HANSER (1980) and REMBOLD and LACKNER (1981) to test the effect of 15 semi-artificial diets on caste differentiation. The aim was to investigate the nutritive conditions for rearing honeybee larvae in vitro into adult pure worker bees. Because the nurse bees feed 4th and 5th instar worker and drone brood on jelly diluted with honey and pollen, we used different diets for young (L2 and L3) and old (L4 and L5) larvae.

The diet for young larvae was a mixture of $50.00 \%$ royal jelly, $36.25 \%$ aqua bidest, $6.25 \%$ fructose, $6.24 \%$ glucose and $1.25 \%$ Difco bacto-yeast extract. In the diets for old larvae the yeast extract was replaced with water.

A stepwise reduction of the royal jelly content in the old larval diet resulted in an increase in mortality. A significant effect on caste differentiation was not observed (Fig. 1). Heat treatment of the royal jelly inhibited the production of queens. More worker bees were reared on such diets (Fig. 2a). Similar results were obtained by feeding larvae on diets without yeast extract (Fig. 2b). This indicates a more pronounced effect of food quality than food quantity on caste development.

A simple and effective method to produce mainly (more than $90 \%$ ) pure workers was found in a uniform supply of the larvae through all feeding stages with diet free of yeast extract. The yield of adult bees was approximately $50 \%$. This feeding program can be applied for in vitro studies of pesticide effects on larval development as well as investigations of brood parasites like the Varroa mite.

It is concluded that in the highly eusocial honeybees a strictly balanced feeding program was evolved only for workers. This enables the colony to produce large numbers of non-fertile individuals on the basis of a minimum quality food. Thus mass rearing of workers became effective with relatively low investment. By any quality improvement, caste differentiation is shifted towards queens. 


\section{LITERATURVERZEICHNIS}

BeEtsma J., 1979. - The process of qucen-worker differenciation in the honeybee. Bee World, 60, 24-39.

Buchner R., 1953. - Beeinflussung der Größe der Arbeitsbiene durch Raum- und Nahrungsmangel während der Larvenzeit. Roux' Arch. Entwicklungsmech., 146, 544-579.

Butenandt A., 1959. - Wirkstoffe des Insektenreiches. Naturwissenschaften, 46, 461-471.

Engels W., 1987. - Reproduction and caste development in social bees. Proc. 10th Intern. IUSSI Congr. München 1986 (in press).

Hanser G., 1980. - Die Aufzucht von Bienenköniginnen im Labor. In : Ruttner F. (ed.), Königinnenzucht. Apimondia-Verlag, Bukarest, Rumänien, pp. 45-64.

Lucht-Bertram E., 1962. - Das postembryonale Wachstum von Hirnteilen bei Apis mellifica L. und Myrmeleon europaeus L. Z. Morphol. Ökol. Tiere, 50, 543-575.

Perez J., 1889. - Les abeilles. Paris, Hachette, $348 \mathrm{p}$.

Planta A.V., 1888. - Über den Futtersaft der Bienen. Hoppe Seyl. Z. physiol. Chemie, 12, 327-354 und 13, 552-561.

Rembold H., 1976. - The role of determination in caste formation in the honey bec. In : Lüscher M. (ed.), Phase and caste determination in insects. Pergamon Press, Oxford, England, pp. 21-34.

Rembold H., LACKNER B., 1981. - Rearing of honeybee larvae in vitro : Effect of yeast extract on queen differentiation. J. Apic. Res., 20, 165-171.

Weiss K., 1978. - Zur Mechanik der Kastenentstehung bei der Honigbiene (Apis mellifica L.). Apidologie, 9, 223-258.

De Wilde J., Beetsma J., 1982. - The physiology of caste development in social insects. Adv. Insect. Physiol., 16, 167-246.

Wittmann D., Engels W., 1981. - Entwicklung von Testverfahren für insektizidbedingte Brutschäden bei der Honigbienc. Mitt. dtsch. Ges. allg. angew. Entomol., 3, 187-190.

Wittmann D., Engels W., Kuhn R., 1987. - Bestimmung der $\mathrm{LD}_{50}$ von Pflanzenschutzmittel an Bienenlarven in vitro mit dem Apis-Larven-Test. Z. angew. Entomol., im Druck. 